\title{
Validation of the Space Weather Modeling Framework using observations from CHAMP and DMSP
}

\author{
H. Wang, ${ }^{1,2}$ A. J. Ridley, ${ }^{1}$ and H. Lühr ${ }^{3}$ \\ Received 17 July 2007; revised 24 November 2007; accepted 17 December 2007; published 6 March 2008.
}

[1] The Space Weather Modeling Framework (SWMF) is a flexible framework for space weather simulation, which can couple magnetosphere and ionosphere processes. This work compares ionospheric outputs from SWMF with magnetic and plasma observations from CHAMP and DMSP satellites under both quiet and storm conditions, emphasizing the dependence of the model's performance for various magnetic local times, solar wind conditions, and seasons. The model predicts the potential better in the dawn-dusk sector then in the noon-midnight sector. For field-aligned currents (FACs) the model performs better on the dayside than on the nightside. In addition, there is a trend toward unsatisfactory behavior in the model as solar activity increases. The model more accurately corresponds to observations during quiet times than disturbed periods. During storms the model FACs tend to locate at $\sim 4^{\circ}$ MLat more poleward than the observations. Our analysis has revealed that the model performance depends strongly on the seasons. The model underestimates the cross polar cap potential (CPCP) by about $~ 50 \%$ in the summer hemisphere while overestimating it by $\sim 50 \%$ in the winter hemisphere. The model calculates the difference between the winter and summer hemisphere CPCP on the order of a factor of 2.5, while DMSP data show that the actual factor is around 1.3. These results reveal that the ionospheric modeling subsets lack appropriate seasonal dependence.

Citation: Wang, H., A. J. Ridley, and H. Lühr (2008), Validation of the Space Weather Modeling Framework using observations from CHAMP and DMSP, Space Weather, 6, S03001, doi:10.1029/2007SW000355.

\section{Introduction}

[2] The near-Earth space environment is quite complex and filled with different electromagnetic structures that may affect technological systems that people rely on [e.g., Sanders, 1961]. Models are attempting to examine these space weather types of effects. A range of models exists from simple empirical relationships that relate geomagnetic conditions in a particular location with solar wind drivers [e.g., Weimer, 1996; Papitashvili and Rich, 2002; Tsyganenko, 2002a, 2002b] to much more sophisticated first-principle models that attempt to solve the entire system driven by solar wind conditions [e.g., Powell et al., 1999; Gombosi et al., 2002; Raeder et al., 2001; Lyon et al., 2004]. Models of the first type are relatively easy to run and do not require much computational power. Models of the second type may be more difficult to work with and often require large computer systems, but their physics

\footnotetext{
${ }^{1}$ Department of Atmospheric, Oceanic and Space Science, University of Michigan, Ann Arbor, Michigan, USA.

${ }^{2} \mathrm{On}$ leave from College of Electronic Informatics, Wuhan University, Hubei, China.

${ }^{3}$ GeoForshungsZentrum Potsdam, Potsdam, Germany.
}

may be more accurate, and they may be able to work outside of the limits of the observations. For example, the linear relationship between the interplanetary magnetic field (IMF) and the ionospheric cross polar cap potential $(\mathrm{CPCP})$ was initially discovered with very limited data sets [e.g., Boyle et al., 1997]. However, later it was shown that the potential saturated for large values of IMF [e.g., Siscoe et al., 2002], which could be easily addressed by MHD models of the magnetosphere [e.g., Ridley, 2007a, 2007b]. Within the last few years near-Earth space models have been coupled together to better represent the physical systems [e.g., Ridley et al., 2001; De Zeeuw et al., 2004; Wang et al., 2004; Wiltberger et al., 2004].

[3] The University of Michigan's Center for Space Environment Modeling (CSEM) has developed the Space Weather Modeling Framework (SWMF), which has the ability to couple numerical domain models flexibly and efficiently, including models of the solar corona, the heliosphere, the magnetosphere, the ionosphere, and the thermosphere [Tóth et al., 2005]. The framework makes the integration, extension, modification, and use of the coupled system more convenient than a single code that would solve a single set of coupled equations. This frame- 
Table 1. Geomagnetic and IMF Conditions During the Simulation Periods of All Six Events

\begin{tabular}{lcccc}
\hline \multicolumn{1}{c}{ Events } & Simulation Time, UT & Minimum $B_{z}, \mathrm{nT}$ & Minimum Dst, $\mathrm{nT}$ & Maximum $A E, \mathrm{nT}$ \\
\hline 31 Mar 2001 & $0200-1400$ & -47.87 & -387 & 1261 \\
17 Apr 2002 & $0800-1800$ & -31.78 & -98 & 1504 \\
4 May 1998 & $0200-1400$ & -37.75 & -205 & 2635 \\
15 Jul 2000 & $1400-2100$ & -57.69 & -198 & 3321 \\
4 Aug 2001 & $1200-2100$ & -5.84 & -17 & 700 \\
20 Nov 2003 & $0900-2100$ & -53.02 & -422 & 2959 \\
\hline
\end{tabular}

work allows for simulations that are not possible with the individual physics models, and it is possible to run them in almost real time on large computer systems. The SWMF used in this work includes the Block-Adaptive Tree Solar Wind Roe-Type Upwind Scheme (BATSRUS) model to simulate the magnetosphere of the Earth [Powell et al., 1999], the Rice Convection Model (RCM) to simulate the inner magnetosphere domain [Toffoletto et al., 2003], and the model described by Ridley et al. [2004] to describe the ionospheric electrodynamics.

[4] Previous validations of these separate domain models exist. For instance, Powell et al. [1999] showed that the numerics of BATSRUS were correct and that grid convergence was achieved. Ridley et al. [2001] compared BATSRUS simulated data to ground-based magnetometer data and found that the MHD simulated magnetic perturbations were much lower than the measured values. Ridley et al. [2002] performed comparisons of BATSRUS simulation results with DMSP data. They showed that the MHD code could reproduce the statistical features of the ionospheric electric potential map. Recently, Tóth et al. [2007] carried out an SWMF simulation of the Halloween storm and compared the ionospheric output with the CPCP estimated from the AMIE technique [Richmond and Kamide, 1988]. They found generally good agreement between these two quantities. This paper is the first that examines how well the coupled model predicts the ionospheric electrodynamic conditions for a series of events.

[5] Since many models are being coupled together, it is crucial to have accurate primary ionospheric quantities. Field-aligned currents (FACs) and ionospheric potential play an important role in the coupling between the magnetosphere and the ionosphere (for more details, see section 2). They are the primary components needed to ensure that the model produces correct results. It is also advisable to examine a wider range of conditions to verify that the code consistently reproduces observations, which is our reason for examining many storms through different seasons.

[6] The SWMF will soon be modified to include a new module for self-consistent ionospheric outflow, an improved radiation belt model, a more realistic auroral specification, a different inner magnetosphere model, and the exchange of more information between modules, which will allow more physical processes to be modeled (e.g., electron heat flux from the inner magnetosphere module to the upper atmosphere module). This study reports the baseline metrics of the ionospheric electrodynamics specification for the current SWMF. In order to run in near real time on 32 nodes, the simulation needs to be low resolution. These low-resolution runs are expected in an operational setting, where speed and robustness outweigh accuracy.

[7] Six events are chosen: one during a quiet period, and five during storm periods, including both equinox and solstice seasons. The selected events allow for a fair assessment of the models. For consistent validation, and in order to quantify the differences between simulations and geophysical measurements, the normalized rootmean-squared (nRMS) difference between the measurements made by satellites and those predicted by the codes is used, similar to the method adopted by Ridley et al. [2002]. The nRMS is defined as

$$
\mathrm{nRMS}=\sqrt{\frac{\sum_{i=1}^{n}\left(y_{i}-y_{i}^{*}\right)^{2}}{\sum_{i=1}^{n}\left(y_{i}^{2}\right)}}
$$

where $y_{\mathrm{i}}$ is the measured quantity and $y_{i}^{*}$ is the model value. A nRMS close to 0 indicates a good model performance. If the model overpredicts the observation, or has an opposite trend, then the nRMS will be larger than 1 .

[8] The geomagnetic and solar wind conditions, like minimum IMF $B_{z}$, minimum $D_{S T}$, and maximum $A E$, for all events are listed in Table 1 . For each event, the model runs for approximately $7 \sim 12 \mathrm{~h}$, covering the storm main phase (see Table 1).

[9] The DMSP satellites cover polar regions at $\sim 835 \mathrm{~km}$ altitude with a fixed local time. The orbit period is approximately $100 \mathrm{~min}$. One of the satellites (F13) has a near dawn-dusk orbit and three $(F 12,14,15)$ have 0930-2130 MLT orbits. The ion drift velocities in the horizontal and vertical direction perpendicular to the satellite track are derived from the ion drift meter (IDM) data [Rich and Hairston, 1994]. The along track potential can be derived from $\Phi=\int(-\vec{V} \times \vec{B}) d s=\int\left(-V_{y} B_{z}\right) d x$, where $\vec{V}$ is the cross track velocity, $\vec{B}$ is the geomagnetic field, $x$ is along the satellite path, $z$ is radially away from the center of the Earth. We neglect $V_{z}$ and $B_{y}$ since they are significantly smaller than $V_{y}$ and $B_{z}$ in the polar region. In this paper, a dipolar magnetic field is used as the $\vec{B}$ field.

[10] The CHAMP satellite was launched on 15 July 2000 into a near-polar $\left(83.7^{\circ}\right.$ incl.) orbit with an initial altitude of $\sim 450 \mathrm{~km}$ [Reigber et al., 2002]. The instrument of prime interest for this study is the fluxgate magnetometer 
(FGM), which delivers vector field readings at a rate of $50 \mathrm{~Hz}$ with a resolution of $0.1 \mathrm{nT}$. The FAC density, $j_{z}$, is determined according to Ampere's law from the magnetic field data by solving the curl- $\mathrm{B}$, that is, $j_{z}=\frac{1}{\mu_{0}}\left(\frac{\partial B_{y}}{\partial x}-\frac{\partial B_{x}}{\partial y}\right)$, where $\mu_{0}$ is the vacuum permeability, and $B_{x}$ and $B_{y}$ are the transverse magnetic field components caused by the currents. We have assumed that the FACs are organized in infinite sheets that are aligned with the mean location of the auroral oval [Wang et al., 2005]. Since we do not have multipoint measurements, we convert observed temporal variations into spatial gradients by considering the velocity, assuming a stationary current, during the time of satellite passage. After discrete sampling is introduced [Lühr et al., 1996], we obtain $j_{z}=\frac{1}{\mu_{0} v_{x}} \frac{\Delta B_{y}}{\Delta t}$, where $v_{x}$ is the velocity perpendicular to the current sheet (i.e., the orbital speed of the satellite) and $B_{y}$ is the magnetic deflection component parallel to the sheet. For the November 2003 event, CHAMP was in the premidnight and prenoon local time sector (2300-1100 MLT), while for the other events, CHAMP was in the early morning and late afternoon local time sector (0300-1500 MLT).

\section{Model Description}

[11] This section describes briefly the SWMF model utilized in this evaluation. It involves domains of global magnetosphere, inner magnetosphere, and ionospheric electrodynamics.

\subsection{Global Magnetosphere}

[12] The global magnetosphere (GM) domain contains the bow shock, magnetopause, and magnetotail of the Earth. The upstream boundary conditions are obtained from ACE satellite measurements. The inner boundary (2.5 $R_{E}$ ) conditions on the velocity are determined by the ionospheric electrodynamics (IE), while the values for the magnetic field and pressure are allowed to float. The number density is set to a constant value of $28 \mathrm{~cm}^{-3}$. The GM component provides FACs to the IE component. FACs are calculated from the curl of the magnetic field at $3.5 R_{E}$, and these are mapped down to the ionosphere. GM is modeled with the BATSRUS code, which is a threedimensional (3-D) global MHD code that has the ability to simulate various plasma environments [Powell et al., 1999]. It has a tilted, rotating dipole, with the rotation axis offset from the dipole axis. The magnetospheric simulations are in GSM coordinates. BATSRUS solves the MHD equations on a block-adaptive Cartesian grid, which allows various scale sizes and discontinuities in the simulated region to be resolved.

[13] For the events under study, BATSRUS has been run using the time-accurate mode and time-varying IMF and solar wind conditions measured by the ACE satellite as inputs at the front boundary. Each event is run with the same resolution, namely, with grid cells that are $1 / 4 R_{E}$ in size in the inner magnetosphere and $1 / 2 R_{E}$ grid cells in the outer magnetosphere. The outer boundaries are placed at $\pm 128 R_{E}$ in the $\mathrm{Y}$ and $\mathrm{Z}$ directions, $+32 R_{E}$ in the upstream direction, and $-224 R_{E}$ in the downstream direction.

\subsection{Inner Magnetosphere}

[14] The inner magnetosphere is modeled with the Rice Convection Model, which describes the drift physics that are not a part of ideal MHD [Wolf et al., 1982; Sazykin et al., 2002]. This code uses the magnetic field topology from GM as well as the electric potential from the Ionospheric Electrodynamics module to advect the different ion energy particles through the inner magnetosphere [De Zeeuw et al., 2004]. RCM is confined to the closed-field region, which is determined by GM. This region can grow and expand according to magnetosphere variations. RCM is solved on a static grid in the ionosphere, which is mapped out to the magnetosphere along field lines produced selfconsistently by GM. The IM module provides pressures to the GM module, which are used to nudge the pressures in BATSRUS over a $20 \mathrm{~s}$ time period. The coupling time between GM and IM is $5 \mathrm{~s}$.

\subsection{Ionospheric Electrodynamics}

[15] The IE domain is a 2-D height integrated spherical surface at normal ionospheric height of about $110 \mathrm{~km}$. It gets FACs from GM. This model is fully described by Ridley et al. [2004]. The solar illumination induced conductivity model used in the present SWMF is from Moen and Brekke [1993]. FACs are used to calculate particle precipitation and conductances, based on a statistical relationship derived from AMIE results, which is $\Sigma=\Sigma_{0} e^{A j_{p}}$, where $\Sigma_{0}$ is a constant conductance and $A$ varies as a function of location and determines whether the current is upward 157 or downward [Ahn et al., 1998]. The potential is solved by $j_{\mathrm{P}}=\nabla_{\perp} \cdot(\Sigma \cdot \nabla \psi)$, where $\Sigma$ is the ionospheric conductance tensor, and $\psi$ is the potential. The potential is then mapped along the magnetic field lines to $2.5 R_{E}$, where the electric field and plasma velocities are derived and used as the inner boundary condition for GM. The coupling time between GM and IE is also $5 \mathrm{~s}$.

\section{Model-Observation Comparison}

[16] This section presents the model-measurement comparison for both FACs and ionospheric potential. The comparison is described separately for magnetically quiet and disturbed periods.

\subsection{Comparison for Quiet Conditions}

[17] A relatively quiet interval chosen by Geospace Environment Modeling (GEM) occurred on 4 August 2001. The model was run between 1200 and 2100 UT, when the solar wind and IMF were relatively steady and strong enough for a moderate CPCP to form. Uncertain- 


\section{Quiet Time Potentials}
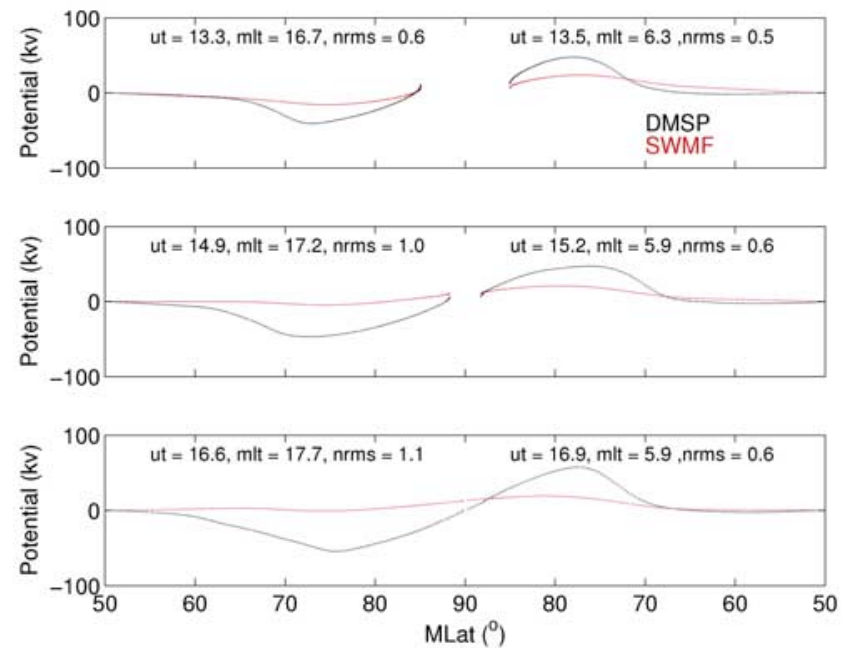

Quiet Time FACs
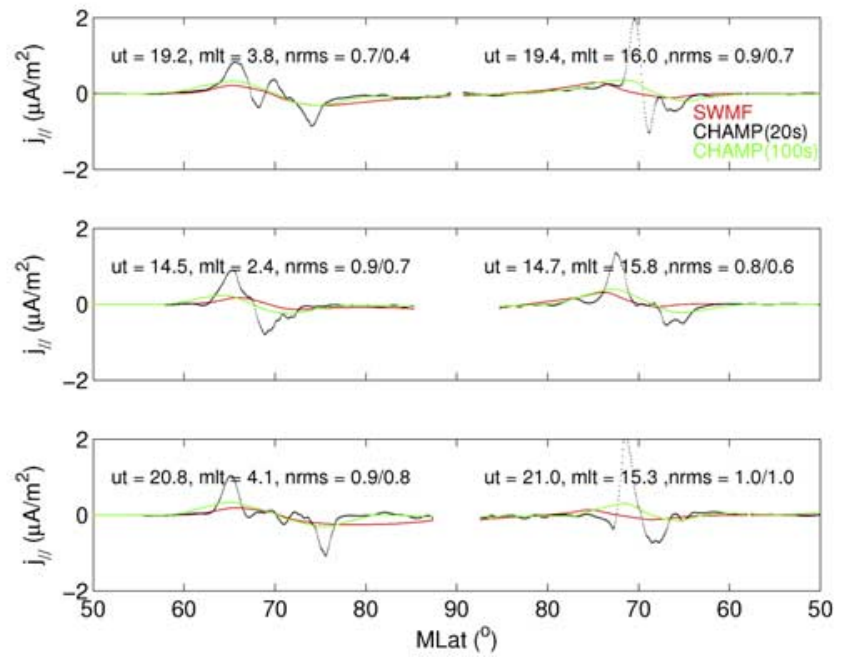

Figure 1. (left) Three examples of the ionospheric potential from SWMF (red) and from DMSP passes (black) across the North Pole during quiet periods, representing from top to bottom the best, average, and worst cases. Also shown is (right) three examples of field-aligned currents, $j_{\mathrm{P}}$, from SWMF (red) and from CHAMP (black and green) during quiet times. Black is CHAMP data filtered with a cutoff period at $20 \mathrm{~s}(150 \mathrm{~km}$ wavelength) and green at $100 \mathrm{~s}$ (760 km wavelength). The listed MLT is valid at the entry of the satellite into the polar region at $65^{\circ}$ MLat.
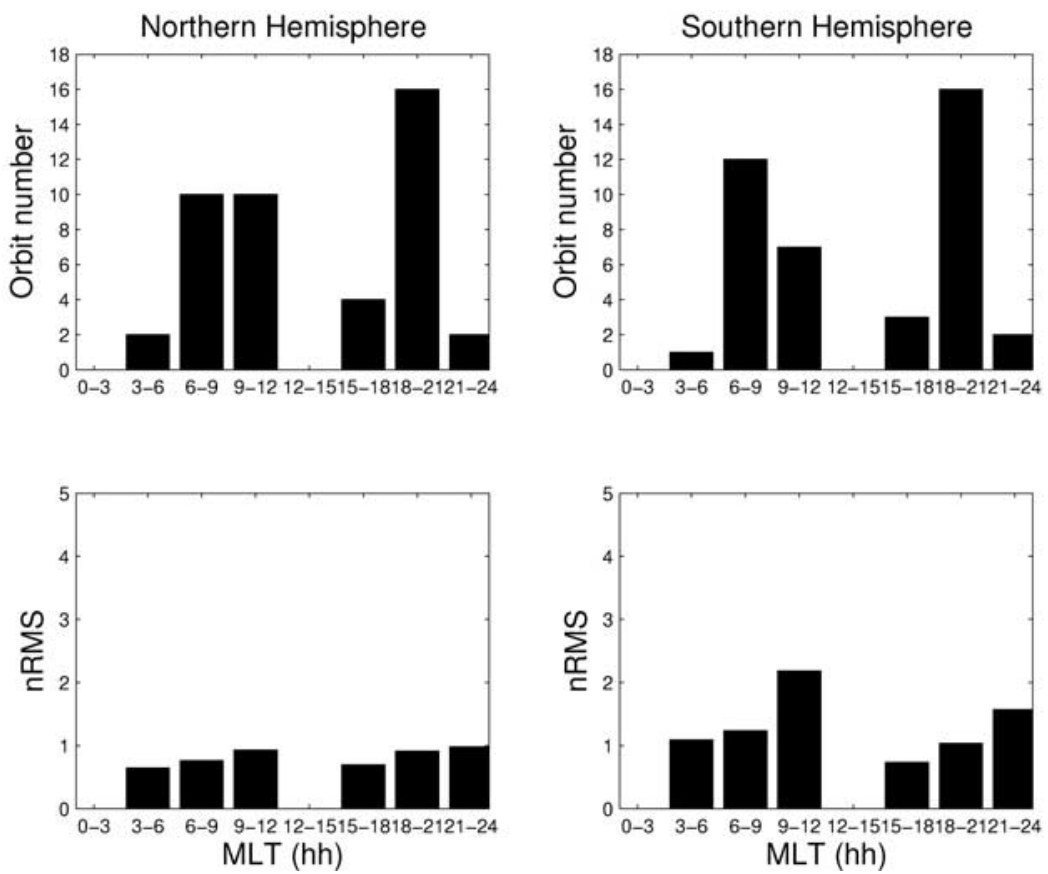

Figure 2. (top) Number of DMSP satellite passes as a function of MLT and (bottom) the nRMS as a function of MLT (left) from the Northern Hemisphere and (right) from the Southern Hemisphere. 

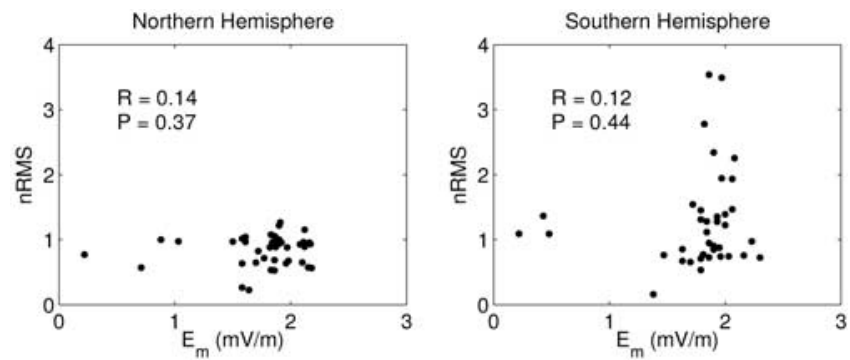

Figure 3. Relation between nRMS and $E_{m}$. Correlation coefficients $\mathrm{R}$ are shown. $\mathrm{P}$ indicates the statistical significance of the correlation. If $\mathrm{P}$ is less than 0.05 , then the correlation $\mathrm{R}$ is significant.

ties in time delays from the ACE satellite should not affect the comparison between this model and observations since the solar wind conditions remained quite steady. A minimum $D_{S T}$ of $-17 \mathrm{nT}$, a minimum $B_{z}$ of $-5.8 \mathrm{nT}$, and a maximum $A E$ of $700 \mathrm{nT}$ were observed during this time interval (see Table 1).

\subsubsection{Ionospheric Potential}

[18] DMSP data from every orbit during the simulation period is compared with the SWMF output. Three sample comparisons over the North Pole are shown in Figure 1 (left), representing the passes that have the best, an average, and the worst nRMS scores from top to bottom. DMSP passed from dusk to dawn (from left to right). The red curve depicts the model output and the black curve represents the satellite measurement. Overall, the SWMF reproduces the observed potential quite well, except for an underestimation of the amplitude. Both red and black curves represent the typical bipolar variations of potential: negative at dusk, positive at dawn. However, the scaling factors between the model and observations are different: the DMSP potential increases from top to bottom, while the model result stays nearly unchanged. For each orbit segment the nRMS is listed in the plots. Normalized RMS errors are smallest on the left orbit at the top and largest at the bottom. The right plots show the field-aligned currents during quiet time periods. These plots will be discussed later.

[19] The model performance appears to have some MLT dependence. To investigate this, the distribution of nRMS
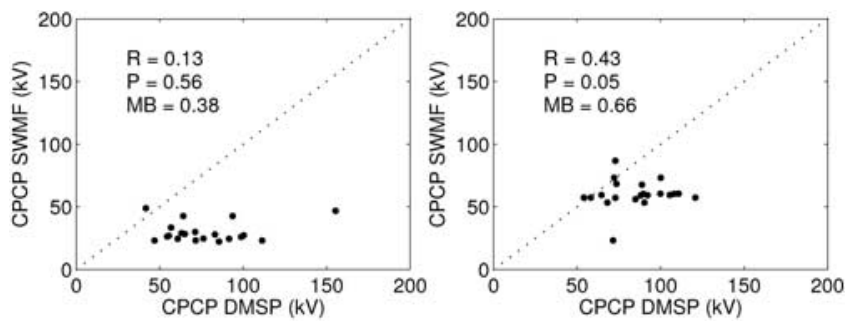

Figure 4. Cross polar cap potential (CPCP) observed by DMSP and predicted by SWMF on 4 August 2001. Correlation parameters $(\mathrm{R}, \mathrm{P})$ are shown in the plots. The multiplicative bias, MB, is also given.
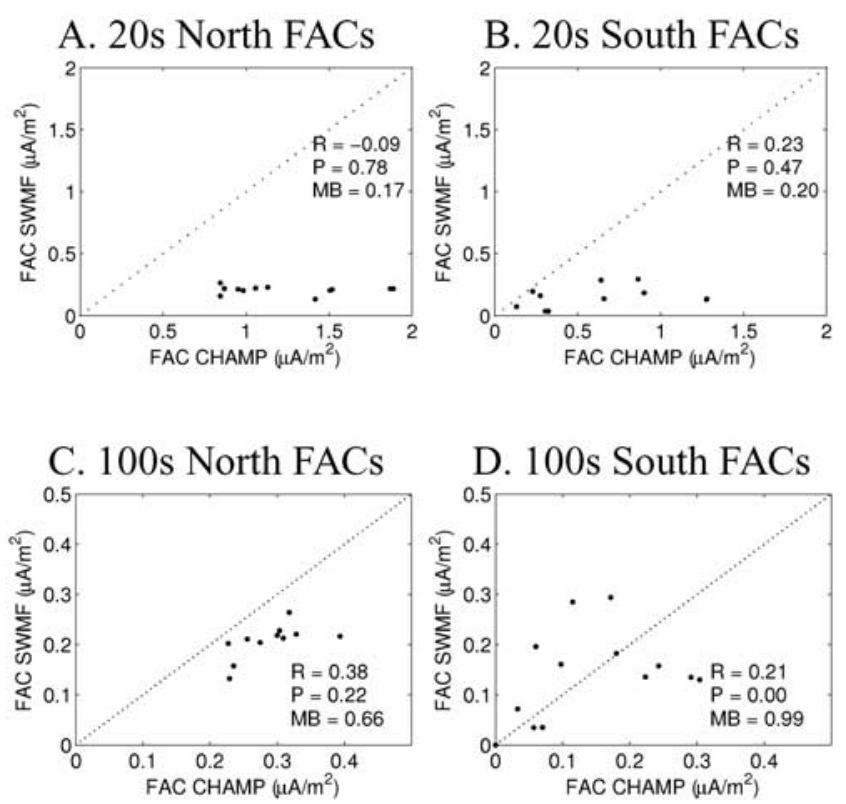

Figure 5. Peak FACs observed by CHAMP and predicted by SWMF on 4 August 2001 in the Northern and Southern Hemispheres. Correlation parameters $(\mathrm{R}, \mathrm{P})$ are shown in together with the multiplicative bias, MB, showing (top) 20-s data and (bottom) 100-s data.

versus MLT is investigated. Figure 2 shows the number of orbits (top) and nRMS (bottom) for eight different MLT sectors in both hemispheres. From the distribution of available orbits, it can be seen that the DMSP satellites cover mainly the $0300-1200$ and 1500-2400 MLT sectors. Larger nRMS errors can be found in the 0900-1200 (prenoon) and 2100-2400 (premidnight) MLT sectors, which suggests that the model has better performance in the dawn-dusk sector than the noon-midnight sector. This applies for both hemispheres. In addition, the nRMS is smaller in the Northern (winter) Hemisphere than in the Southern (summer).

[20] The solar wind conditions have an important effect on the ionospheric potential [e.g., Reiff et al., 1981]. Therefore we would like to see how the model performs under different solar wind conditions. In this study, we use the merging electric field, $E_{m}=v_{s w} \sqrt{B_{y}^{2}+B_{z}^{2}} \sin ^{2}(\theta / 2)$ [e.g., Kan and Lee, 1979], where $v_{s w}$ is the solar wind velocity and $\theta$ is the clock angle of the IMF defined in GSM coordinates, as the geoeffective solar wind electric field part. Subsequently, it is considered as a measure for the solar wind input to the magnetosphere, since previous studies have shown that $E_{m}$ is a suitable geoeffective parameter [e.g., Troshichev and Lukianova, 1996]. The solar wind and IMF data are measured by the Advanced Composition Explorer (ACE) satellite, which is located at the Lagrange point (L1) approximately $220 R_{E}(\sim 1.48$ million $\mathrm{km})$ in front of the Earth. 
Active Time Potentials
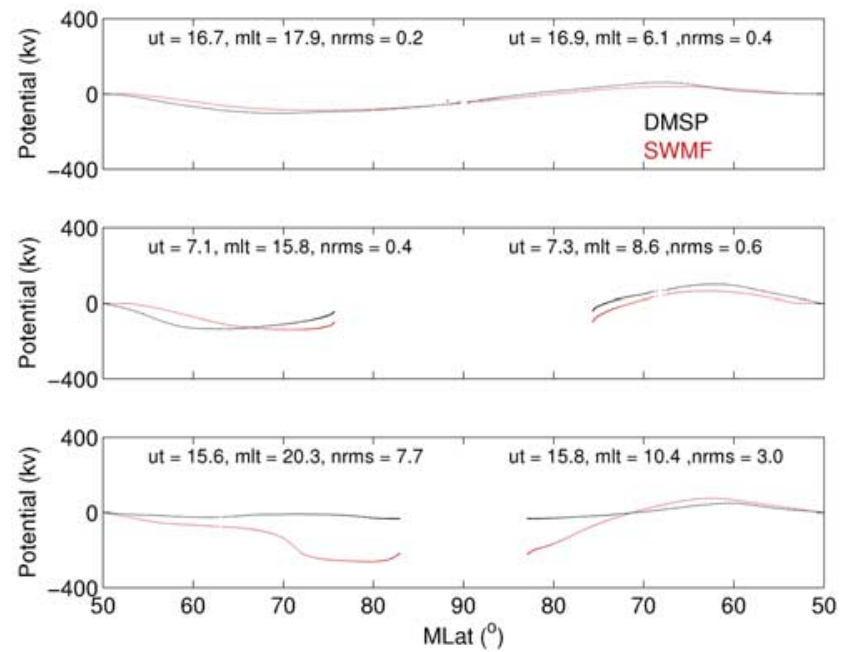

Active Time FACs
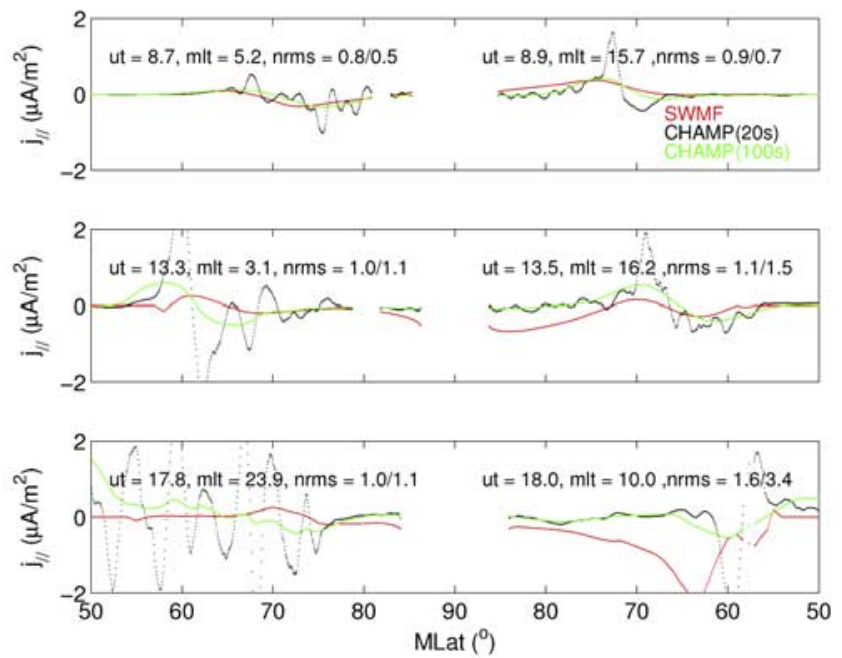

Figure 6. (left) Three examples of the ionospheric potential from SWMF (red) and from DMSP (black) across the North Pole during storm periods, representing the best, average, and worst cases for the runs from top to bottom. Also shown is (right) three examples of the field-aligned currents, $j_{\mathrm{P}}$, from SWMF (red) and from CHAMP (black and green) during storm times. Black is CHAMP data filtered with a cutoff period at $20 \mathrm{~s}(150 \mathrm{~km}$ wavelength) and green at $100 \mathrm{~s}(760 \mathrm{~km}$ wavelength). The listed MLT refers to the entry of the satellite into the polar region at $65^{\circ}$ MLat.

The transit time to the magnetopause of each event was computed individually using the actual solar wind speed data. Figure 3 shows the relation of nRMS and $E_{m}$ in a scatterplot. The nRMS varies a great deal around $E_{m}=2 \mathrm{mV} / \mathrm{m}$. One can see that the nRMS
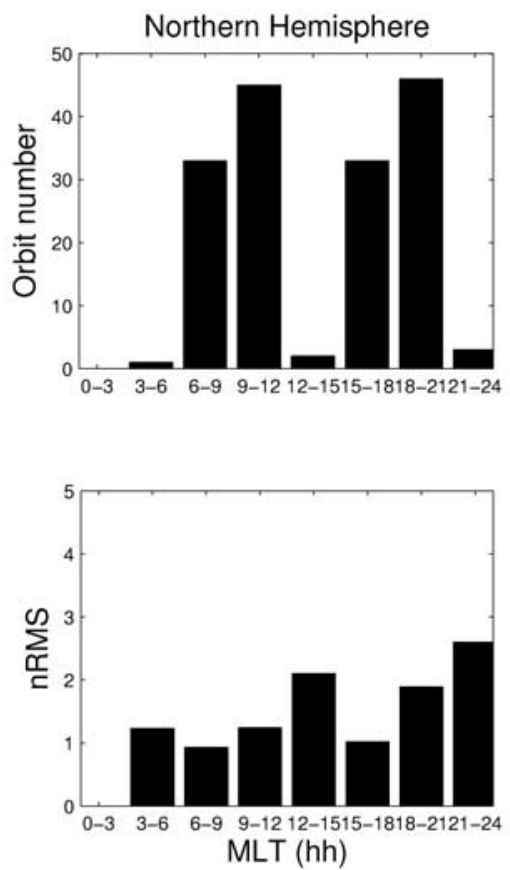

errors tend to increase as $E_{m}$ increase, with larger values in the Southern Hemisphere as compared to the Northern Hemisphere. We have also recorded the peak potential and CPCP for each orbit and performed a correlation analysis with respect to $E_{m}$, separately for
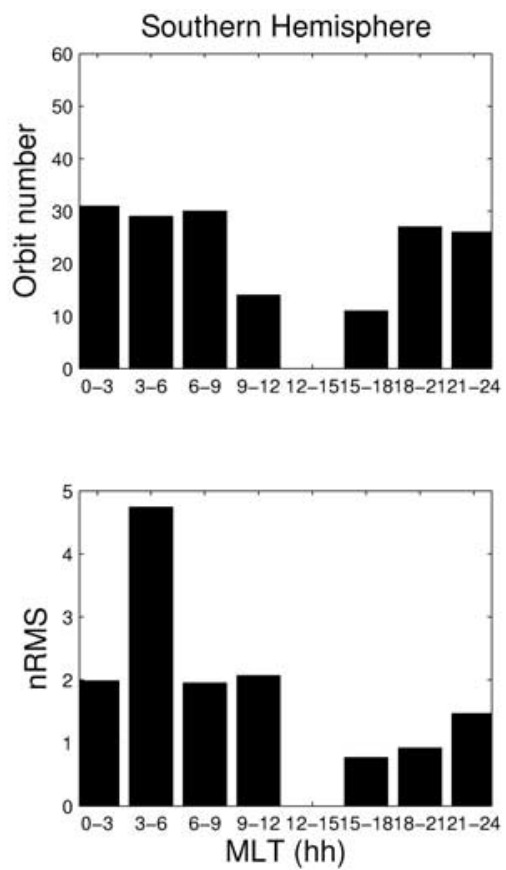

Figure 7. Number of DMSP satellite passes, and nRMS comparison with DMSP potentials in the same format as Figure 2 except for storm time. 

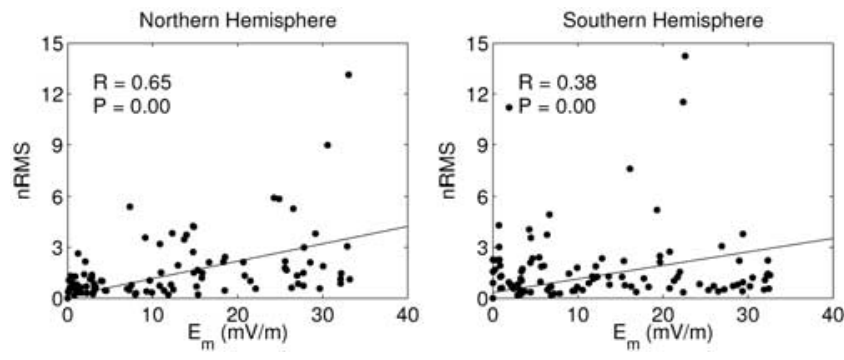

Figure 8. Relation between nRMS and $E_{\mathrm{m}}$ in the same format as Figure 3 except for storm time.

the model data and observation (not shown). However, no useful correlations are found between them.

[21] To determine whether the SWMF systematically underestimates or overestimates the $\mathrm{CPCP}$, a scatterplot of the CPCP predicted by the SWMF versus those observed by DMSP for both hemispheres is shown in Figure 4. The multiplicative bias, $\mathrm{MB}=\Sigma(\mathrm{CPCP} \mathrm{SWMF}) /$ $\Sigma($ CPCP DMSP), has also been calculated for each of the events, which might be informative for the general space weather community. The model results are smaller than the DMSP measurements in both hemispheres. Previous studies also reported that the CPCP derived from AMIE was lower than that observed by DMSP [Kihn et al., 2006].
In addition, the difference between the winter CPCP and summer $\mathrm{CPCP}$, derived from SWMF, is on the order of a factor of 2. However, the DMSP data show that the factor is around 1.

\subsubsection{Field-Aligned Currents}

[22] Three examples of comparisons between FACs observed by CHAMP and derived by SWMF over the North Pole are shown in Figure 1 (right) when CHAMP passed from midnight to noon (from left to right), representing the best, the average, and the worst passes from top to bottom. The red curve depicts the model output and the black curve depicts the CHAMP data filtered with a 20-s cutoff period, while the green line indicates the CHAMP data with a filter of $100 \mathrm{~s}$. There are dominant FACs seen in the 20-s filtered data (150 km wavelength), which disappear when a filter at $100-\mathrm{s}$ (750 km wavelength) is applied. The classical dual-sheet pattern remains in both. There is better agreement between the model and the 100-s filtered data in both topology and amplitude. In correspondence to that, the nRMS are also smaller. The normalized RMS errors are smallest on the left orbit section at the top and largest on the right at the bottom. We prefer to use 20-s averaged data since they were more typically used in previous studies [e.g., Papitashvili and Rich, 2002; Wang et al., 2005], so that direct comparisons to those study results are possible. However, 100-s averaged data are
A. May 5, 1998 N.H.
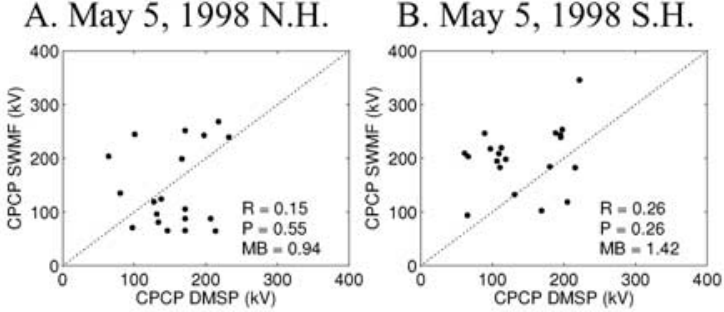

C. July 15,2000 N.H.

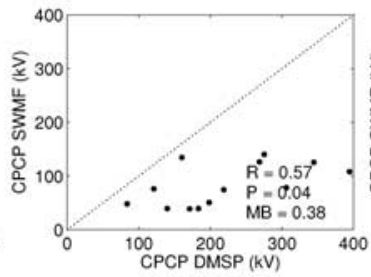

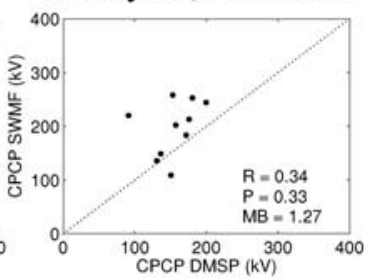

E. March 31, 2001 N.H. F. March 31, 2001 S.H.
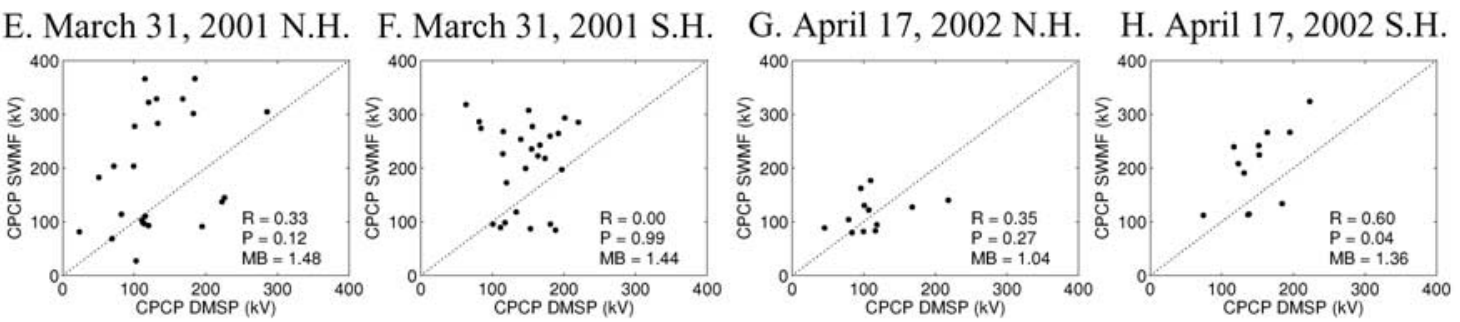

I. Nov. 20, 2003 N.H.

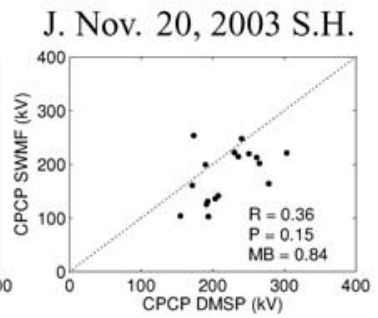

Figure 9. Cross polar cap potential (CPCP) observed by DMSP and predicted by SWMF for all storm events. Correlation parameters $(\mathrm{R}, \mathrm{P})$ and multiplicative bias $(\mathrm{MB})$ are shown in the plots. 

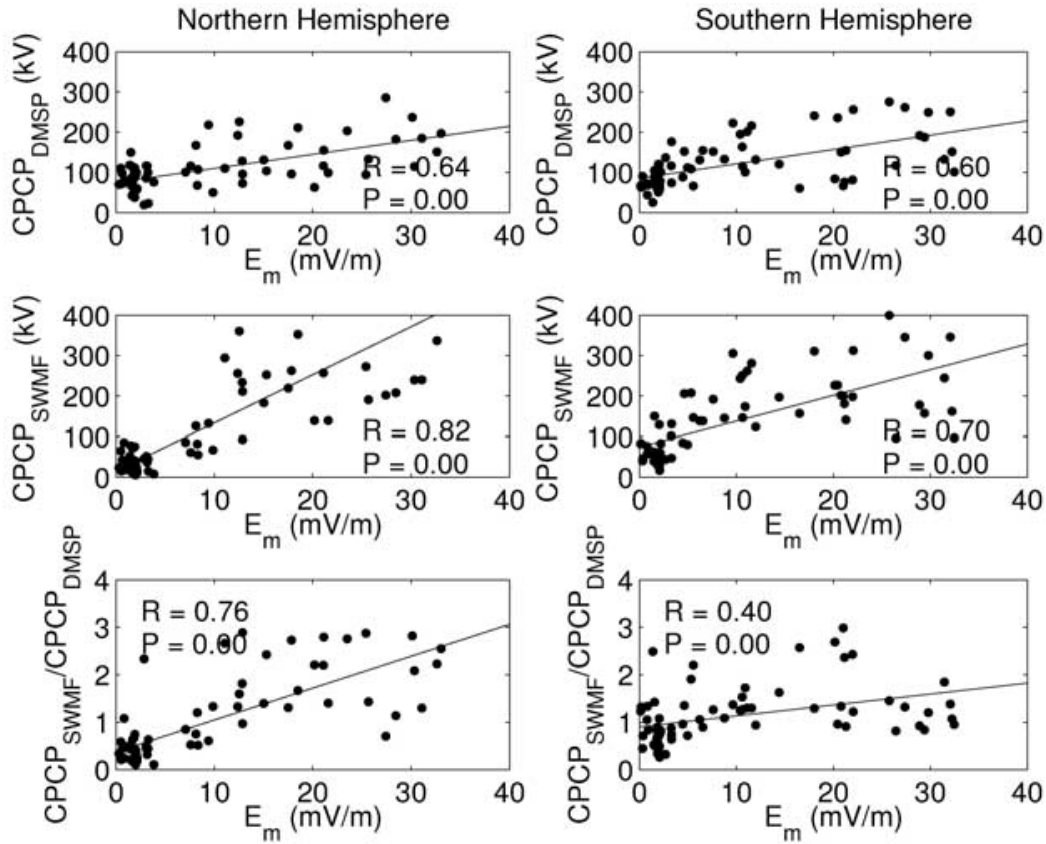

Figure 10. CPCP from (top) DMSP, (middle) SWMF, and (bottom) the ratios between the model and observation, as a function of $E_{m}(\mathrm{left})$ in the Northern Hemisphere and (right) in the Southern Hemisphere. Correlation parameters $(\mathrm{R}, \mathrm{P})$ are shown.

A. 20s North FACs

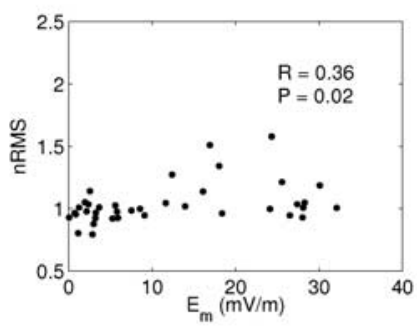

C. 100 s North FACs

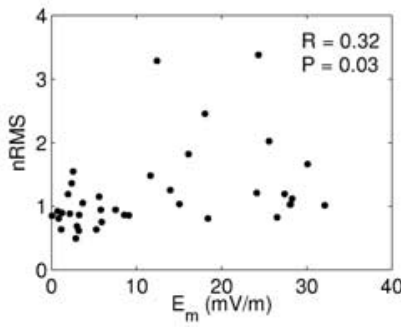

B. 20s South FACs

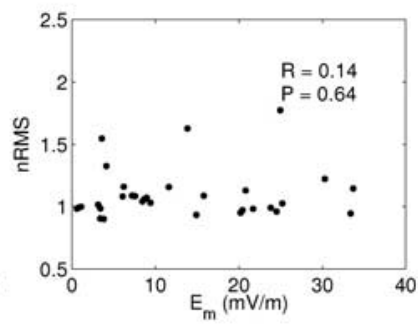

D. 100 s South FACs

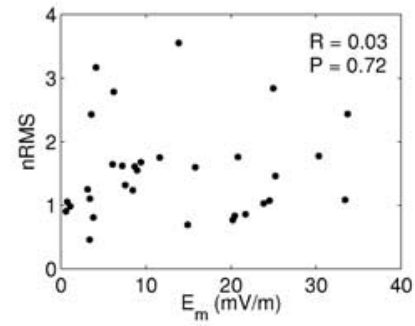

Figure 11. Relation between nRMS and $E_{m}$ for FAC parameters in the Northern and Southern Hemispheres. Correlation parameters $(\mathrm{R}, \mathrm{P})$ are shown in the plots. Shown are $(\mathrm{a}, \mathrm{b}) 20$-s data and (c, d) 100-s data. 

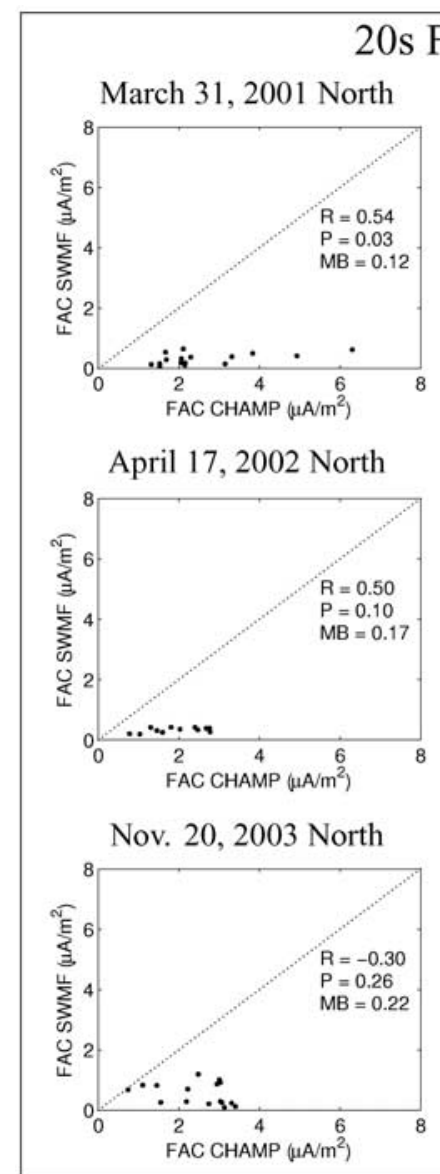

100s FACs

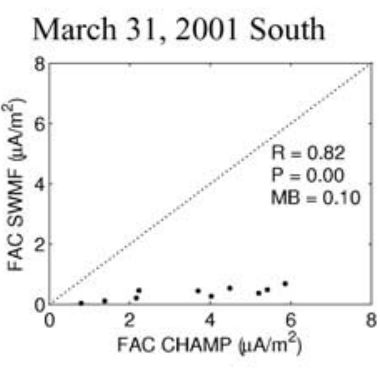

April 17, 2002 South

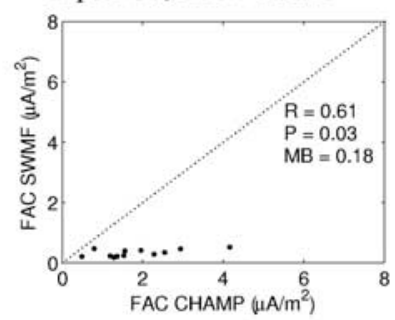

Nov. 20, 2003 South

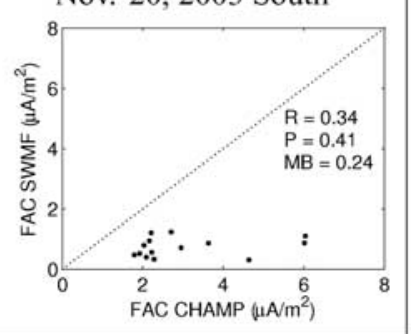

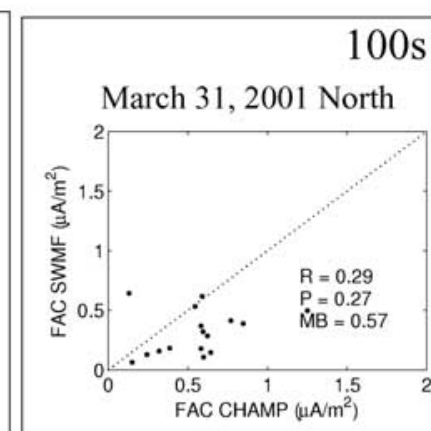

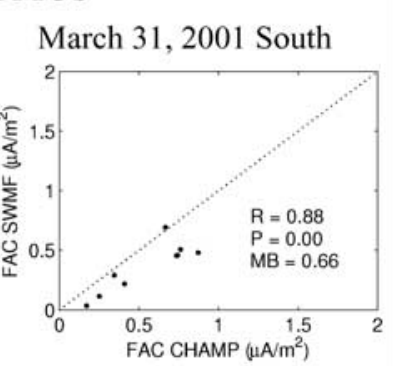

April 17, 2002 North

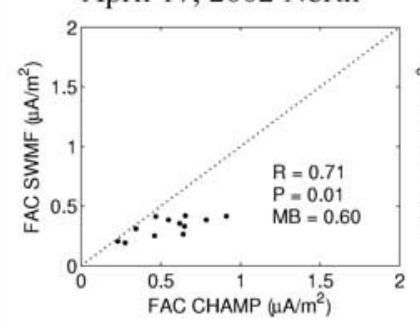

Nov. 20, 2003 North

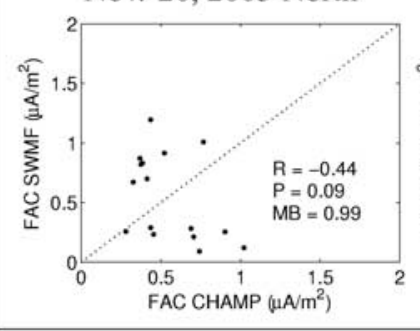

April 17, 2002 South

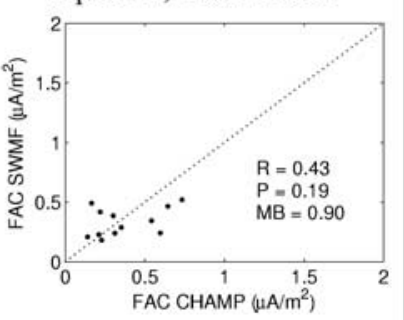

Nov. 20, 2003 South

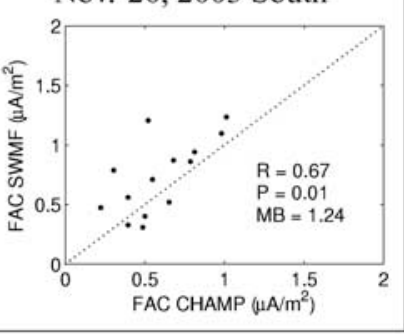

Figure 12. Field-aligned currents predicted by SWMF and observed by CHAMP for the three storm events in both hemispheres. Correlation parameters $(R, P)$ and multiplicative bias $(M B)$ are shown, with (left) 20-s data and (right) 100-s data.

presented to show that the model has the overall structure accurate on a very gross scale. Except for special statements, the following results are described for the 20-s case.

[23] We have compared the model's early morning performance to that of late afternoon local time sectors (not shown). The model performs better on the dayside than the nightside and better in the Northern Hemisphere than in the Southern Hemisphere. The dependence of the model performance on solar activity is also investigated (not shown). It appears that the nRMS tends to increase as $E_{m}$ increases, which is more obvious in the Southern Hemisphere than in the Northern Hemisphere. However, the correlations between these two parameters are weak.

[24] In order to get a more quantitative impression of the relation between the FAC density predicted by SWMF and that observed by CHAMP, a correlation analysis is performed. Figure 5 shows the relation of the peak-topeak values of these two quantities in a scatterplot for both the North Pole and the South Pole. The multiplicative bias, $\mathrm{MB}=\Sigma($ FAC SWMF $) / \Sigma($ FAC CHAMP $)$, have also been calculated for each of the events, which might be infor- mative for the general space weather community. The SWMF underestimates the current density by a factor of $\sim 4(2.5)$ in the Northern (Southern) Hemisphere. In addition, the difference between the summer and winter hemisphere FACs densities of SWMF is on the order of a factor of $\sim 1.3$, while CHAMP data shows that the factor is $\sim 2$. From the 100-s filtered data, it can be seen that the model predictions are much closer to the observations.

\subsection{Comparison for Disturbed Conditions}

[25] In this section, we describe the comparison of the model to the observations during five magnetic storms with a minimum $D_{S T}$ ranging from -98 to $-422 \mathrm{nT}$, minimum $B_{z}$ from -31 to $-58 \mathrm{nT}$, and maximum $A E$ from 1261 to $3321 \mathrm{nT}$ (see Table 1). This represents more of a challenge for the model since the inner magnetospheric dynamics should dominate the geospace environment.

\subsubsection{Ionospheric Potential}

[26] Three sample comparisons over the North Pole during storm periods are shown in Figure 6 (left), representing the best, the average, and the worst passes. The 

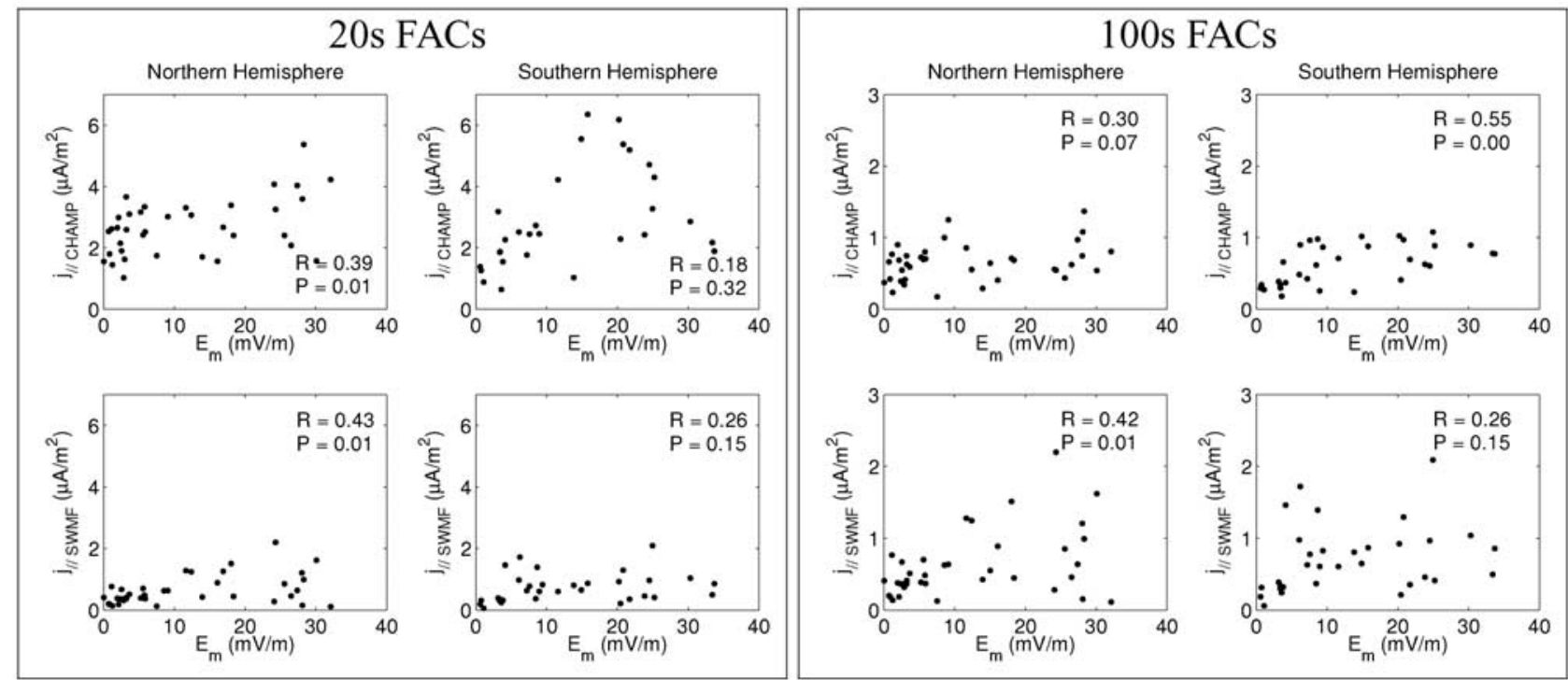

Figure 13. Peak FACs, $j_{\mathrm{P}}$, from (top) CHAMP and (bottom) SWMF as a function of $E_{m}$ in the Northern and Southern Hemispheres. Correlation parameters (R, P) are shown, with (left) 20-s data and (right) 100-s data.

model agrees relatively well with the observations for the first two cases but deviates from the observations for the last pass.

[27] Figure 7 shows the local time distribution of the number of orbits (top) and nRMS (bottom). In the Northern Hemisphere orbits are primarily in the 0600-1200 and 1500-2100 MLT sectors. Similar to those during quiet period, larger nRMS errors are found in the 0900-1200 MLT (prenoon) and 1800-2100 (premidnight) sectors. In the Southern Hemisphere more events are in the $0000-$ 1200 and 1500-2400 MLT with larger nRMS errors in the 0000-1200 MLT (morning) sector. Generally, nRMS values are smaller in the Northern Hemisphere than in the Southern Hemisphere.

[28] The solar wind coupling effect on the model's performance is also investigated. Figure 8 shows the nRMS values as a function of $E_{m}$ in both hemispheres. In the Northern Hemisphere a reasonable correlation can be found with $\mathrm{R}=0.65$, and a linear relationship nRMS = $0.11^{*} E_{m}$ is derived. In the Southern Hemisphere the scatter is large, and therefore the result is not as significant. Nevertheless, from the regression line, on average, the model results are deviating from observations as the solar wind activity is becoming larger.

[29] Figure 9 shows the CPCP observed by DMSP and calculated from SWMF separately for the Northern Hemisphere and Southern Hemisphere for all storm events. The correlation coefficients and multiplicative bias have also been shown for each of the events. It is interesting to note from these plots that there is a strong bias in the results between the summer and winter hemispheres, in which the CPCP from the model is stronger in the winter and weaker in the summer as compared to the observa- tions. Enhanced scatter between the model and the observation can be found at equinox. In addition, the difference between the winter hemisphere CPCP and the summer hemisphere CPCP from SWMF is on the order of a factor of $1.5 \sim 3$. However, DMSP data shows that the factor is around $1 \sim 1.5$.

[30] To investigate whether the SWMF has systematic deficiencies in the determination of the CPCP, the modeled and measured potentials are compared with the merging electric field. Figure 10 shows plots of the CPCP from DMSP (top) and SWMF (middle), versus $E_{m}$. There are reasonably good correlations with $R>0.6$ emerging in all four cases. In both hemispheres and for both the model and the observations, the CPCP tends to increase as $E_{m}$ increases. The rate of increment is larger in the model than in the observation. In the Northern Hemisphere, the intercept of the regression line is different from model to observation. In the Southern Hemisphere, both model and observation have nearly the same intercept. In addition to the general trend being relatively correct, it is also interesting to notice that in the Southern Hemisphere, there is a saturation of the CPCP for $E_{m}$ larger than $10 \mathrm{mV} / \mathrm{m}$, which can be observed in both model and satellite results. However, this saturation does not occur in the Northern Hemisphere. The same analysis has been performed with other parameters such as peak potentials and locations of the peak potential, but none of them shows a good correlation with $E_{m}$. The dependence of the ratios between SWMF and DMSP CPCP on $E_{m}$ is shown at the bottom of Figure 10. It is found that there is a fairly good correlation with $\mathrm{R}=0.76$ between the ratio and $E_{m}$ in the Northern Hemisphere. When $E_{m}<10 \mathrm{mV} / \mathrm{m}$, the model result is smaller than observations. However, when $E_{m}$ is larger 

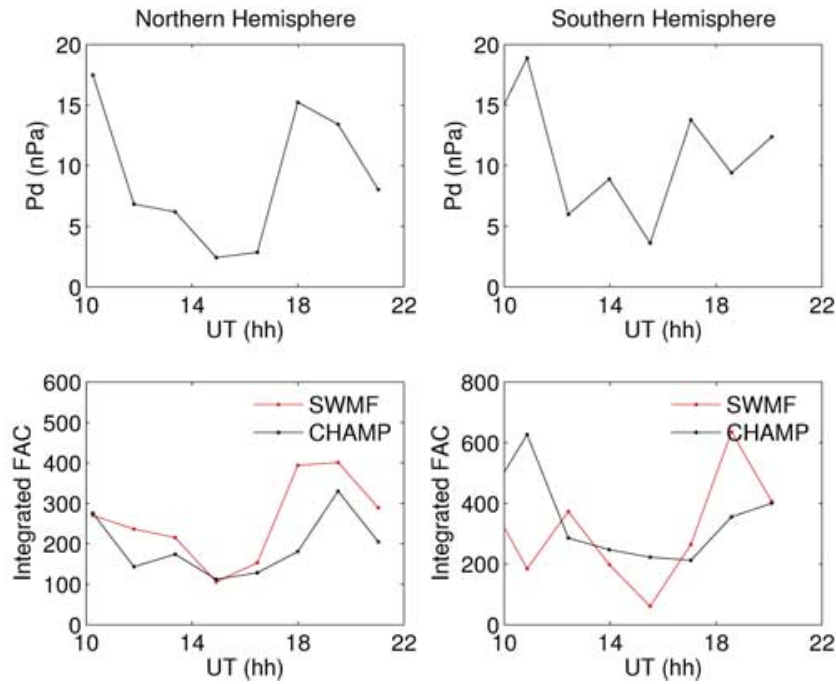

Figure 14. Storm time $P_{d}$ and integrated field-aligned currents along the orbit segments predicted by SWMF and observed by CHAMP in the daytime sector on 20 November 2003.

than $10 \mathrm{mV} / \mathrm{m}$, the model result becomes much larger than observations by a factor of 2 or 3.

\subsubsection{Field-Aligned Currents}

[31] Three CHAMP examples passes during storm periods over the North Pole are shown in Figure 6 (right) when CHAMP passes from the nightside to the dayside (from left to right). They represent the best, the average, and the worst passes. During storm periods FACs on the dayside are well-shaped but on the nightside more complicated structures form, with multisheets covering a wide range of latitudes. The latter cannot be reproduced by the model.

[32] CHAMP data are available for three storm events in the noon-midnight sectors. The results show that the model performs better on the dayside than the nightside, and the model works better in the Northern Hemisphere than the Southern Hemisphere (figures not shown). Figure 11 shows nRMS values derived from the comparison of FAC densities as a function of $E_{m}$. It seems that nRMS values tend to slightly increase as $E_{m}$ increases. The local time, hemispheric, and solar wind activity dependence during storm periods are all similar to those during the quiet period for the FACs. In the left plots most of the points cluster around one, which shows that the SWMF currents are significantly lower than the CHAMP currents. In the right plots (i.e., 100s averages) there are many points far away from one, which indicates that the model result has the correct order of magnitude as compared to the CHAMP derived FACs.

[33] Figure 12 shows the peak FACs predicted by SWMF versus those observed by CHAMP for three storms. The correlation coefficients and multiplicative bias are shown in the plots. The SWMF current densities are always smaller than the 20-s averaged CHAMP derived current densities. Relatively good correlation can be found for the equinox storms. In addition, the difference between the summer and winter hemisphere FAC densities from SWMF is on the order of a factor of $\sim 1$, while CHAMP data indicates that the factor is $\sim 1.5$.

[34] The merging electric field has an important effect on FACs [Wang et al., 2005]. Figure 13 shows the maximum FACs density, $j_{\mathrm{P}}$, from CHAMP (top) and SWMF (bottom) as a function of $E_{m}$. Unfortunately, poor correlations are found for them. However, it can be seen that $j_{\mathrm{p}}$ tends to increase as $E_{m}$ increases, which is the same for both the model and the observation. The saturation effect for $E_{m}>10 \mathrm{mV} / \mathrm{m}$ is quite evident for CHAMP data filtered at 100s. SWMF shows the saturation also, but it is not as clear as in the data. Previous studies have shown that during storms, the largest FACs densities have no one-to-one correspondence with the largest solar wind input, characterized by $B_{\mathrm{z}}$ or $E_{m}$, etc. However, solar wind dynamic pressure, $P_{d}$, was found to have a good correlation with the FACs strength on the dayside during a storm [Wang et $a l ., 2006]$. In order to test whether the model reflects this relation, $P_{d}$ and integrated FACs predicted by the SWMF and observed by CHAMP in the daytime sector on
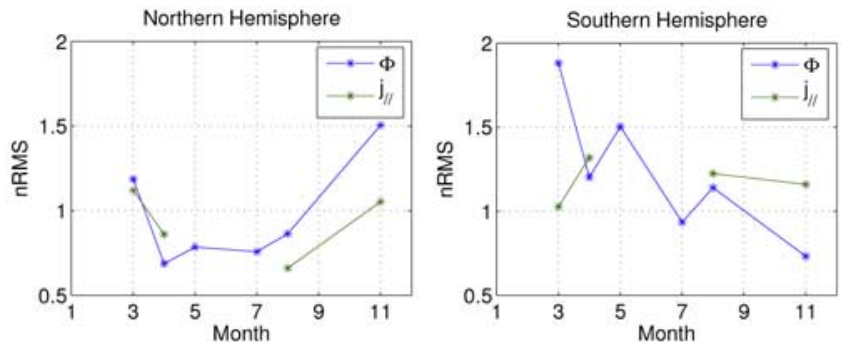

Figure 15. Averaged nRMS of ionospheric potential, $\Phi$, and FACs, $j_{\mathrm{P}}$, for all events in the (left) Northern Hemisphere and (right) Southern Hemisphere. 

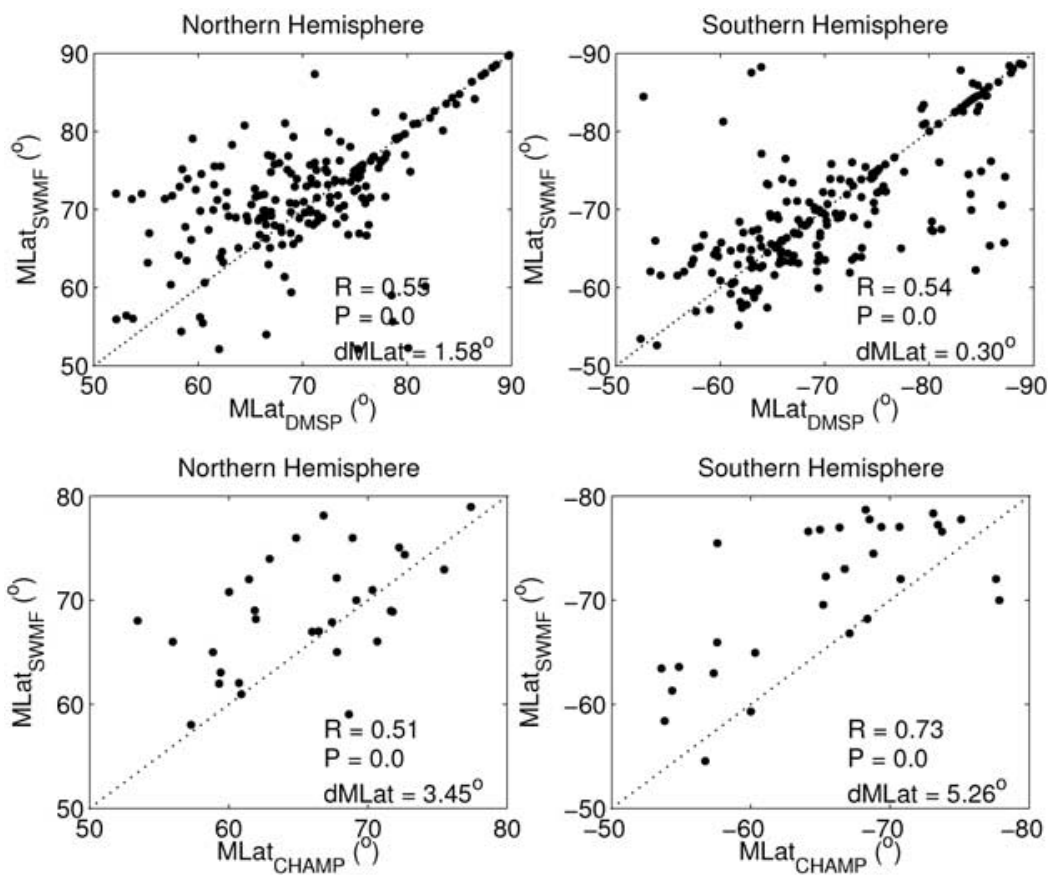

Figure 16. (top) Scatterplots of the MLat of the peak potential from SWMF versus DMSP, from (left) the Northern Hemisphere and (right) the Southern Hemisphere, and (bottom) the MLat of peak FACs from CHAMP versus SWMF.

20 November 2003 are plotted in Figure 14. The time series of integrated FACs are determined by summing up the absolute FAC densities from a current-free location $\left(40^{\circ}\right.$ MLat employed in this study) to the highest latitude of each crossing. The integration includes both the negative and positive FAC densities and thus represents the total FAC in each segment of the orbit. There are some small changes in the solar wind input conditions between CHAMP's passages of the polar region in the two hemispheres, which are separated by about $30 \mathrm{~min}$. Both the modeled and observed FACs strength have a good correlation with $P_{d}$ in both hemispheres. The same analysis has been performed with the other two storm events on 31 March 2001 and 17 April 2002, but neither of them shows good correspondences between $P_{d}$ and FACs strength even in the data, thus suggesting there must be other factors also affecting FACs strength.

\subsection{Seasonal Effects}

[35] As a summary of CHAMP and DMSP comparisons, we have produced nRMS errors for all of the events. For simplification, we have averaged nRMS values for each parameter over the simulation time for each event. Figure 15 shows the averaged nRMS as a function of month for all events in the Northern (left) and Southern (right) Hemispheres. The nRMS of potentials and currents in the Northern Hemisphere are smaller than those in the Southern Hemisphere at equinox, while the summer hemispheric nRMS values are typically smaller than the winter hemispheric nRMS values.

\section{Discussion}

[36] The purpose of this paper is to compare SWMF simulation results with measurements of the currents and potential in the ionosphere, which may help users of the SWMF code to understand its limitations when running at relatively low resolution and close to real time. The SWMF couples models of several different domains together. For this coupling system, it is especially important to have the primary components correctly reproduced. For this purpose we have selected FACs and ionospheric potential to compare with high resolution CHAMP and DMSP observations. The potential distribution is derived from the FACs and a conductivity model. The shortages in FACs will, of course, propagate into the СРСР. Several typical events are chosen ranging from quiet to storm periods. In general, the comparisons reveal that the model can capture the large-scale feature of ionospheric potential and FACs. In particular, we are concentrating on three primary points related to the model performances: (1) MLT dependence; (2) solar wind input effect; (3) seasonal effect.

\subsection{Local Time Dependence}

[37] One of the interesting conclusions to note from this part of study is that the nRMS values have some MLT dependence. For the ionospheric potential the model 
performs better in the dawn-dusk sector compared to the noon-midnight sector. This is consistent with previous studies [Kihn et al., 2006], in which they compared AMIE with DMSP CPCP data and found that the comparison with the F13 satellite was better than the other satellites. Several reasons have been proposed for the discrepancy between the dawn-dusk and noon-midnight meridian: (1) fewer errors introduced by minimizing $V_{x}$ (i.e., the along-track component of the ion flow) for the dawn-dusk orbit because it cuts through the convections cell in a more perpendicular direction than the other spacecrafts; (2) the contribution from light contamination within the IDM sensor is smaller in the dawn-dusk meridian compared to the noon-midnight orbits. This light contamination can cause spurious high-speed horizontal sunward flow measurements, which drive the potential anomalies; (3) if the model is slightly rotated by $\delta \alpha$ with respect to the actual pattern, then the error is proportional to $\sin (\delta \alpha)$ along the noon-midnight satellite track. Since the F13 orbit (i.e., dawn-dusk) is sampling closer to the heart of the pattern, a slight rotation has a far less dramatic effect (error proportional to $\cos (\delta \alpha)$ ) leading to a better correlation with the observations.

[38] For FACs estimates, unfortunately, we do not have access to other spacecraft in the dawn-dusk orbit. For all the considered events, CHAMP is flying in the noonmidnight sector. It reveals that the model performs better on the dayside compared to the nightside. This is not surprising because during storms a well-defined FAC sheet can be found on the dayside in both hemispheres, while there are multiple FAC sheets on the nightside, covering a wide range of latitudes (about $21^{\circ}$ )[Wang et al., 2006]. The model is unable to capture the smaller-scale (fine) structure of FACs due to its coarse resolution. In addition, the model cannot produce currents equatorward of $55^{\circ}$ MLat due to the limitation of its inner boundary. The dayside FACs are observed by CHAMP to be located at higher-latitude compared to the nightside [e.g., Wang et al., 2006], so it is more probable that nightside currents will move inside of the inner boundary of the code. Finally, the model does not capture substorm features very well, which are localized mainly on the nightside. During substorms the magnetospheric and the ionospheric conditions are significantly altered. The change of one or some of these conditions should cause the variations of the current system on the nightside. These factors can explain why the model is not functioning as well on the nightside as it is on the dayside.

[39] Another point that should be mentioned is that a general uncertainty remains, inherent to FAC estimates based on a single spacecraft. We have assumed that the FACs are organized in infinite sheets that are aligned with the mean location of the auroral oval. Evidence for such geometry has been examined previously [e.g., Iijima and Potemra, 1976; Sugiura and Potemra, 1976]. During magnetic storms these conditions may not apply. However, Lühr et al. [1996] showed that any deviation (oblique crossing or finite extent of sheet) can lead to an underestimation of the current density. The latitude of the peak current density is, however, not significantly affected by uncertainty in the current geometry and can be regarded as reliable. Since the model tends to predict FACs densities much lower than CHAMP observed values, we can state that the SWMF model underestimates the FACs (see Figure 5 and 12). And the absolute value of current density is not crucial in this validation study. A previous study also found that the BATSRUS simulated ionospheric Hall currents were much lower than observations [Ridley et al., 2001]. It should be noted that with $1 / 4 R_{E}$ resolution at $3.5 R_{E}$, the sample points in the ionosphere are separated by $170 \mathrm{~km}$. For a full wave, at least two samples are needed. This determines, basically, the maximum resolution of the FAC that can be resolved by the MHD code. Because of diffusion, one would expect these to be even more smooth, which is what we show here. When the CHAMP measurements are filtered at $100 \mathrm{~s}$ (which corresponds to a $750 \mathrm{~km}$ wavelength), we obtain better agreement with the model results (see Figures 1 and 6). With a higher resolution in the MHD code, the FACs would be more confined and may have a larger magnitude.

\subsection{Solar Wind Input Effects}

[40] The SWMF shows that the CPCP is linearly related to $E_{m}$ with a reasonable correlation and that the trend is relatively consistent with measurements. In addition to the general correct trend, it is also found that the CPCP may be saturating when $E_{m}$ is larger than $10 \mathrm{mV} / \mathrm{m}$. This has been shown in both the model and the actual data, suggesting the model can match the physical trends. Previous studies have shown that there are nonlinear developments of the CPCP under extreme solar wind conditions [e.g., Ridley, 2005; Nagatsuma, 2002]. The mechanisms responsible for the saturation are still under debate. Some have interpreted the saturation as an internal mechanism, caused by R1 currents reducing the strength of the magnetic field near the magnetopause, thus limiting the amount of reconnection [e.g., Siscoe et al., 2002]. Others described it as an external mechanism because the ionospheric CPCP could be modeled quite well by an empirical relationship with the limiting amplified field [Reiff et al., 1981] or by including the solar wind Mach number in that empirical relationship [Ridley, 2005]. Interestingly, both the model and the data show indications of saturation for the events described here, but the saturation is occurring in one hemisphere only. This indicates that the ionospheric conductivity may play a role in the saturation and should be examined more closely [Nagatsuma, 2002].

[41] We have also studied the relationship of the locations of ionospheric parameters between the model and data. Figure 16 (top) shows the MLat of peak potentials derived from SWMF versus those observed by DMSP. The model tends to reside $\sim 1.6^{\circ}\left(0.3^{\circ}\right)$ MLat (on average) poleward of the potential peak measured by DMSP in the Northern (Southern) Hemisphere. Figure 16 (bottom) 
shows MLat of peak FACs from SWMF versus the data. On average, the model resides $\sim 4^{\circ}$ MLat poleward of measurements. The location of the maximum in the ionosphere potential and FAC density derived from SWMF suggests a polar cap boundary several degrees poleward of that observed by satellites during storm periods. Partially, this can be attributed to the inner boundary of the model $\left(3.5 R_{E}\right)$, corresponding to $55^{\circ}$ MLat in the ionosphere. FACs at lower latitude cannot be reproduced. However, during severe storms FACs are observed to expand equatorward as low as $50^{\circ}$ MLat [Wang et al., 2006], which is far below the inner boundary of the model.

[42] Previous studies have proposed a number of different methods to make the model currents shift more equatorward but all have disadvantages [e.g., Ridley et al., 2001]. For example, one is to move the inner boundary of the model closer to Earth. However, this would cause the wave speed to increase and reduce the time step of the simulation. Another might be to increase the model's resolution in the inner boundary. Either of these solutions will cause the model to run much slower, a trade off which must be made in order to maintain accuracy. Other methods include coupling the MHD code to a more sophisticated inner magnetosphere model [e.g., Liemohn et al., 2001] or including artificial, simplistic, R2 currents [Ridley et al., 2002]. These may allow the equatorward part of FACs to be more accurately modeled, since the morphology of this part of FACs is affected by the plasma sheet and ring current [Zheng et al., 2006].

\subsection{Seasonal Effects}

[43] Subsequently, we have examined possible seasonal effects in the model-data comparison. Figure 9 shows a clear difference in the CPCP between the summer and winter comparisons, in which SWMF underestimates the CPCP by about $\sim 50 \%$ in summer but overestimates it by about $\sim 50 \%$ in winter. Figure 12 shows that the modeled peak FAC densities are $\sim 38 \%$ of observation in summer and $\sim 23 \%$ in winter. Thus the conductances used in the model, including solar illumination induced and particle precipitation induced parts, seem to be much smaller than observations, especially in winter.

[44] The model calculates a difference between the winter and summer hemisphere CPCP on the order of a factor of 2.5, while DMSP data show that the true factor is around 1.3 (see Figure 9). On the other hand, the observed FACs change by approximately a factor of 1.5 from summer to winter, while the model FAC densities stay almost the same (see Figure 12). Thus the conductivity has to change by a factor of 2.5 from summer to winter in the model while 1.95 in the data. Previous statistical studies reported that the difference between the winter CPCP and summer hemisphere CPCP is around $1.1 \sim 1.15$ [Papitashvili and Rich, 2002], and the difference between the summer FACs and winter FACs is around $1.5 \sim 2$ [Wang et al., 2005]. Then the conductivity in the summer hemisphere is assumed to be larger by a factor of $1.7 \sim 2.4$ than in the winter hemisphere. These ranges are comparable to our satellite observations during storms, and they suggest that seasonal differences of both conductivity and potential in the model are too large while those of FACs are too small. In addition, the model performs relatively better in summer than in winter (see Figure 15). This seasonal discrepancy suggests that the ionospheric electrodynamics model lacks seasonal dependence in the conductivity [Ridley, 2007a, $2007 \mathrm{~b}]$. The reliability of the relation presented here should be verified on a statistical basis.

[45] A large percentage of the runs are failing to match the observations (nRMS > 1), especially during storm periods (see Figures 8 and 11). Using $E_{m}$ as a measure for the solar wind input, we see in Figures 8 and 11 that there is a trend toward unsatisfactory behavior in the model as solar activity increases. It is also shown in Figure 10 that when $E_{m}$ is larger than $10 \mathrm{mV} / \mathrm{m}$, the model overestimates the CPCP compared to observations by a factor of 2 or 3 . There are several factors that may account for the too large CPCP during storm periods. One is that the reconnection rate on the dayside in the SWMF is too high as southward $B_{\mathrm{z}}$ increases toward large values. This would cause the CPCP to become too large. The second possible mechanism is the lack of significant R2 currents. Owing to the absence of R2 currents, more R1 currents have to close through the cross polar cap Pedersen current, which causes the CPCP increases [De Zeeuw et al., 2004]. The third factor is ionospheric conductivity, which may be incorrectly reproduced, especially during storm periods. Previous studies showed that with higher conductivity, one could get lower potential [Ridley et al., 2004]. The verification of the conductivity model will be addressed in more detail in the ongoing work. We will try to improve the conductivity model used in the present SWMF model by considering the empirical models [e.g., Brekke and Hall, 1988; Brekke et al., 1989] and the radar (e.g., Millstone Hill and EISCAT) and satellite (e.g., IMAGE and DMSP) observations.

\section{Summary}

[46] A detailed validation study of ionospheric outputs from the SWMF has been performed. By comparing with CHAMP and DMSP observations obtained in both the Northern and Southern Hemispheres for a series of events, the capability of the SWMF to model several crucial parameters has been evaluated. This is the first study investigating how well the SWMF specifies the ionospheric electrodynamic conditions for a series of events. The results are summarized as follows:

[47] 1. The model reproduces the ionospheric potential better in the dawn-dusk sector than in the noon-midnight sector and reproduces FACs better on the dayside than on the nightside.

[48] 2. The SWMF reproduces a significant correlation between the predicted CPCP and its solar wind input, represented by the merging electric field, $E_{m}$. It is also found in both, data and model, that the CPCP may 
saturate at high $E_{m}$ values $(>10 \mathrm{mV} / \mathrm{m})$. This indicates that the model can get the general physical trends correctly. The ratio between SWMF and DMSP CPCP has a fairly good correlation with $E_{m}$. When $E_{m}<10 \mathrm{mV} / \mathrm{m}$, the model result is smaller than observations. However, when $E_{m}$ is larger than $10 \mathrm{mV} / \mathrm{m}$, the model result becomes much larger than the observation, even by a factor of 2 or 3 . There seems to be a dependence of the model's performance on solar wind input. A trend toward unsatisfactory behavior occurs as activity levels increase. Thus the model has a relatively better performance during quiet times than during disturbed periods.

[49] 3. The magnetic latitudes of peak FACs derived from SWMF are on average $4^{\circ}$ MLat poleward of those observed by satellites, while the peak locations of the potentials are at approximately the same location as the observation.

[50] 4. The model underestimates FAC densities in both hemispheres. Both modeled and observed FAC strengths seem to correlate well with the solar wind dynamic pressure. When the measured FACs are averaged over approximately $750 \mathrm{~km}$, the agreement improves significantly.

[51] 5. The modeled CPCP is $\sim 50 \%$ larger in the winter hemisphere and $\sim 50 \%$ smaller in the summer hemisphere than the observations. On average, the differences between the winter and summer hemisphere CPCP is on the order of a factor of 2.5, while DMSP data show that the true factor is around 1.3. Observed FACs vary by approximately a factor of 1.5 from summer to winter, while SWMF predictions stay almost unchanged.

[52] 6. There is a dependence of the model's performance on season. The model matches the data better in the summer than in the winter. These results may suggest that the ionospheric modeling subsets lack seasonal dependence.

[53] Acknowledgments. We are obliged to F. Christiansen of DNSC for kindly providing the ACE solar wind data propagated to the magnetopause. We thank ACE teams for providing solar wind and IMF data. The Center for Space Sciences at the University of Texas at Dallas is acknowledged for providing the DMSP drift velocity data. The operational and data processing supports of the CHAMP mission by the German DLR and BMBF are greatly appreciated. This work is supported by the NASA research grant F010639, NSF grant ATM0639336, and by National Nature Science Foundation of China (40604017).

\section{References}

Ahn, B.-H., A. D. Richmond, Y. Kamide, H. W. Kroehl, B. A. Emery, O. de la Beaujardiére, and S.-I. Akasofu (1998), An ionospheric conductance model based on ground magnetic disturbance data, J. Geophys. Res., 103, 14,769-14,780.

Boyle, C. B., P. H. Reiff, and M. R. Hairston (1997), Empirical polar cap potentials, J. Geophys. Res., 102, 111-126.

Brekke, A., and C. Hall (1988), Auroral ionospheric quiet summer time conductances, Ann. Geophys., 6, 361-375.
Brekke, A., C. Hall, and T. L. Hansen (1989), Auroral ionospheric conductances during disturbed conditions, Ann. Geophys., 7, $269-280$.

De Zeeuw, D. L., S. Sazykin, R. A. Wolf, T. I. Gombosi, A. J. Ridley, and G. Tóth (2004), Coupling of a global MHD code and an inner magnetospheric model: Initial results, J. Geophys. Res., 109, A12219, doi:10.1029/2003JA010366.

Gombosi, T. I., G. Tóth, D. L. D. Zeeuw, K. C. Hansen, K. Kabin, and K. G. Powell (2002), Semirelativistic magnetohydrodynamics and physics-based convergence acceleration, J. Comput. Phys., 177, 176205.

Iijima, T., and T. Potemra (1976), Field-aligned currents in the dayside cusp observed by Triad, J. Geophys. Res., 81, 5971-5979.

Kan, J. R., and L. C. Lee (1979), Energy coupling function and solar wind-magnetosphere dynamo, Geophys. Res. Lett., 6, 577-580.

Kihn, E. A., R. Redmon, A. J. Ridley, and M. R. Hairston (2006), A statistical comparison of the AMIE derived and DMSP-SSIES observed high-latitude ionospheric electric field, J. Geophys. Res., 111, A08303, doi:10.1029/2005JA011310.

Liemohn, M. W., J. U. Kozyra, M. F. Thomsen, J. L. Roeder, G. Lu, J. E. Borovsky, and T. E. Cayton (2001), Dominant role of the asymmetric ring current in producing the stormtime Dst* J. Geophys. Res., 106, $10,883-10,904$.

Lühr, H., J. Warnecke, and M. K. A. Rother (1996), An algorithm for estimating field-aligned currents from single spacecraft magnetic field measurements: A diagnostic tool applied to Freja satellite data, IEEE Trans. Geosci. Remote Sens., 34, 1369-1376.

Lyon, J. G., J. A. Fedder, and C. M. Mobarry (2004), The Lyon-FedderMobarry (LFM) global MHD magnetospheric simulation code, J. Atmos. Sol. Terr. Phys., 66, 1333-1350.

Moen, J., and A. Brekke (1993), The solar flux influence on quiet time conductances in the auroral ionosphere, Geophys. Res. Lett. 20, 971-974.

Nagatsuma, T. (2002), Saturation of polar cap potential by intense solar wind electric fields, Geophys. Res. Lett., 29(10), 1422, doi:10.1029/2001GL014202.

Papitashvili, V. O., and F. J. Rich (2002), High-latitude ionospheric convection models derived from Defense Meteorological Satellite Program ion drift observations and parameterized by the interplanetary magnetic field strength and direction, J. Geophys. Res., 107(A8), 1198, doi:10.1029/2001JA000264.

Powell, K. G., P. L. Roe, T. J. Linde, T. I. Gombosi, and D. L. de Zeeuw (1999), A solution adaptive upwind scheme for ideal magnetohydrodynamics, J. Comput. Phys., 154, 284-309.

Raeder, J., R. L. McPherron, L. A. Frank, S. Kokubun, G. Lu, T. Mukai, W. R. Paterson, J. B. Sigwarth, H. J. Singer, and J. A. Slavin (2001), Global simulation of the Geospace Environment Modeling substorm challenge event, J. Geophys. Res., 106, 381-395.

Reiff, P. H., R. W. Spiro, and T. W. Hill (1981), Dependence of polar cap potential drop on interplanetary parameters, J. Geophys. Res., 86, $7639-7648$.

Reigber, C., H. Lühr, and P. Schwintzer (2002), CHAMP mission status, Adv. Space Res., 30, 129-134.

Rich, F. J., and M. Hairston (1994), Large-scale convection patterns observed by DMSP, J. Geophys. Res., 99, 3827-3844.

Richmond, A. D., and Y. Kamide (1988), Mapping electrodynamic features of the high-latitude ionosphere from localized observations: Technique, J. Geophys. Res., 93, 5741-5759.

Ridley, A. J. (2005), A new formulation for the ionospheric cross polar cap potential including saturation effects, Ann. Geophys. $23,3533-3547$

Ridley, A. J. (2007a), The effects of seasonal changes in the ionospheric conductances on magnetospheric field-aligned currents, Geophys. Res. Lett., 34, L05101, doi:10.1029/2006GL028444.

Ridley, A. J. (2007b), Alfvén wings at Earth's magnetosphere under strong interplanetary magnetic fields, Ann. Geophys., 25, 533-542.

Ridley, A. J., D. L. De Zeeuw, T. I. Gombosi, and K. G. Powell (2001), Using steady state MHD results to predict the global state of the magnetosphere ionosphere system, J. Geophys. Res., 106, 30,06730,076 . 
Ridley, A. J., K. C. Hansen, G. Tóth, D. L. De Zeeuw, T. I. Gombosi, and K. G. Powell (2002), University of Michigan MHD results of the Geospace Global Circulation Model metrics challenge, J. Geophys. Res., 107(A10), 1290, doi:10.1029/2001JA000253.

Ridley, A. J., T. I. Gombosi, and D. L. De Zeeuw (2004), Ionospheric control of the magnetosphere: Conductance, Ann. Geophys., 22, $567-584$.

Sanders, R. (1961), Effect of terrestrial electromagnetic storms on wireline communications, IRE Trans. Commun. Syst., 9, 367-377.

Sazykin, S., R. A. Wolf, R. W. Spiro, T. I. Gombosi, D. L. D. Zeeuw, and M. F. Thomsen (2002), Interchange instability in the inner magnetosphere associated with geosynchronous particle flux decreases, Geophys. Res. Lett., 29(10), 1448, doi:10.1029/2001GL014416.

Siscoe, G. L., G. M. Erickson, B. U. O. Sonnerup, N. C. Maynard, J. A. Schoendorf, K. D. Siebert, D. R. Weimer, W. W. White, and G. R. Wilson (2002), Hill model of transpolar potential saturation: Comparisons with MHD simulations, J. Geophys. Res., 107(A6), 1075, doi:10.1029/2001JA000109.

Sugiura, M., and T. A. Potemra (1976), Net field-aligned currents observed by Triad, J. Geophys. Res., 81, 2155-2164.

Toffoletto, F., S. Sazykin, R. Spiro, and R. Wolf (2003), Inner magnetospheric modeling with the Rice Convection Model, Space Sci. Rev., $107,175-196$.

Tóth, G., et al. (2005), Space Weather Modeling Framework: A new tool for the space science community, J. Geophys. Res., 110, A12226, doi:10.1029/2005JA011126.

Tóth, G., D. Zeeuw, T. I. Gombosi, W. B. Manchester, A. J. Ridley, I. V. Sokolov, and I. I. Roussev (2007), Sun-to-thermosphere simulation of the 28-30 October 2003 storm with the Space Weather Modeling Framework, Space Weather, 5, S06003, doi:10.1029/2006SW000272.

Troshichev, O. A., and R. Y. Lukianova (1996), Polar cap index (PC) as a proxy for ionospheric electric field in the near-pole region, Geophys. Res. Lett., 27, 3809-3812.

Tsyganenko, N. A. (2002a), A model of the near magnetosphere with a dawn-dusk asymmetry: 1 . Mathematical structure, J. Geophys. Res., 107(A8), 1179, doi:10.1029/2001JA000219.
Tsyganenko, N. A. (2002b), A model of the near magnetosphere with a dawn-dusk asymmetry: 2. Parameterization and fitting to observations, J. Geophys. Res., 107(A8), 1176, doi:10.1029/2001JA000220.

Wang, H., H. Lühr, and S. Y. Ma (2005), Solar zenith angle and merging electric field control of field-aligned currents: A statistical study of the southern hemisphere, J. Geophys. Res., 110, A03306, doi:10.1029/2004JA010530.

Wang, H., H. Lühr, S. Y. Ma, J. Weygand, and R. M. Skoug (2006), Field-aligned currents observed by CHAMP during the intense 2003 geomagnetic storm events, Ann. Geophys., 24, 311-324.

Wang, W., M. Wiltberger, A. G. Burns, S. C. Solomon, T. L. Killeen, N. Maruyama, and J. G. Lyon (2004), Initial results from the coupled magnetosphere-ionosphere-thermosphere model: Thermosphereionosphere responses, J. Atmos. Terr. Phys., 66, $1425-1441$, doi:10.1016/j.jastp.2004.04.008.

Weimer, D. R. (1996), A flexible, IMF dependent model of high-latitude electric potentials having "space weather" applications, Geophys. Res. Lett., 23, 2549-2552.

Wiltberger, M., W. Wang, A. G. Burns, S. C. Solomon, J. G. Lyon, and C. C. Goodrich (2004), Initial results from the coupled magnetosphere ionosphere thermosphere model: Magnetospheric and ionospheric responses, J. Atmos. Terr. Phys., 66, $1411--1423$, doi:10.1016/j.jastp.2004.03.026.

Wolf, R. A., M. Harel, R. W. Spiro, G. Voigt, P. H. Reiff, and C. K. Chen (1982), Computer simulation of inner magnetospheric dynamics for the magnetic storm of July 29, 1977, J. Geophys. Res., 87, 5949-5962.

Zheng, Y., A. T. Y. Lui, M. C. Fok, B. J. Anderson, P. C. Brandt, T. J. Immel, and D. G. Mitchell (2006), The relationship between Region 2 field-aligned current and the ring current: Model results, J. Geophys. Res., 111, A11S06, doi:10.1029/2006JA011603.

H. Lühr, GeoForshungsZentrum Potsdam, D-14473 Potsdam, Germany.

A. J. Ridley and H. Wang, Department of Atmospheric, Oceanic, and Space Science, University of Michigan, Ann Arbor, MI 48109, USA. (ridley@umich.edu) 